\title{
Indicadores de avaliação da Insegurança Alimentar e Nutricional e fatores associados: revisão sistemática
}

\author{
Evaluation indicators of Food and Nutritional Insecurity and \\ associated factors: systematic review
}

Dayane de Castro Morais (https://orcid.org/0000-0001-6439-7009) ${ }^{1}$

Sílvia Oliveira Lopes (https://orcid.org/0000-0002-6755-8610) ${ }^{1}$

Silvia Eloíza Priore (https://orcid.org/0000-0003-0656-1485) ${ }^{1}$

${ }^{1}$ Departamento de Nutrição e Saúde, Centro de Ciências Biológicas e da Saúde, Universidade Federal de Viçosa. Av. P. H. Rolfs s/n, Centro. 36570-000 Viçosa MG Brasil.

dayanecm@yahoo.com.br

\begin{abstract}
The scope of this paper was to systematically investigate the indicators used in the evaluation of food and nutritional insecurity and associated factors in Brazilian studies. After selection in the databases using search terms and reverse search, 89 articles were included. The majority of the articles evaluated food and nutritional insecurity according to the indicator of perception, using the Brazilian Scale of Food Insecurity (83.1\%), the American Scale (5.62\%) and isolated questions $(2.25 \%)$. The other articles evaluated the presence of malnutrition among children (1.12\%), expenditure on food (1.12\%), adult-equivalent dietary intake (1.12\%), protocols $(2.25 \%)$, and prediction models (2.25\%). Food and nutritional insecurity (measured by the different indicators) was associated with socioeconomic and demographic factors, health and lifestyle conditions, food consumption and nutritional status, based on families, specific age groups, beneficiaries of government programs, among others, as sample units. The majority of the studies merely evaluated the food dimension of the insecurity, thereby increasing the difficulty in evaluating this complex situation.
\end{abstract}

Key words Food and nutritional security, Indicators, Methodologies
Resumo Objetivou-se investigar sistematicamente os indicadores utilizados na avaliação da insegurança alimentar e nutricional e os fatores associados, em estudos brasileiros. Após seleção nas bases de dados e busca reversa, utilizando os termos de busca, incluiu-se 89 artigos. A maioria destes avaliou a insegurança segundo indicador de percepção, utilizando Escala Brasileira de Insegurança Alimentar (83,1\%), Escala Americana $(5,62 \%)$ e perguntas isoladas $(2,25 \%)$. Os demais avaliaram pela presença de desnutrição em crianças (1,12\%), gastos com alimentação $(1,12 \%)$, consumo alimentar de adulto equivalente $(1,12 \%)$, protocolos $(2,25 \%)$ e modelos de predição (2,25\%). A insegurança alimentar e nutricional, mensurada pelos distintos indicadores, associou-se a fatores socioeconômicos e demográficos, condiçôes de saúde e estilo de vida, consumo alimentar e estado nutricional, tendo como unidade amostral famílias, grupos etários específicos, beneficiários de programas governamentais, entre outros. A maioria dos estudos avaliou apenas a dimensão alimentar da insegurança, reforçando a dificuldade de avaliação desta complexa situação. Palavras-chave Segurança alimentar e nutricional, Indicadores, Metodologias 


\section{Introdução}

A avaliação da Insegurança Alimentar e Nutricional (IAN) deve pautar-se na utilização de múltiplos indicadores, que abarquem as dimensões de acesso, disponibilidade, utilização biológica e estabilidade. Estes indicadores devem contemplar o amplo conceito de Segurança Alimentar e Nutricional (SAN), adotado no país, de garantia de acesso a alimentos em qualidade e quantidade suficiente, sem comprometer as demais necessidades essenciais ${ }^{1-3}$.

Dentre os indicadores de Insegurança Alimentar (IA) e/ou nutricional comumente adotados, tem-se as escalas de percepção de fome, a disponibilidade calórica, o consumo alimentar, o estado nutricional e os fatores socioeconômicos. Escalas de percepção, como a Escala Brasileira de Insegurança Alimentar (EBIA), são indicadores diretos de avaliação da IA, porém não mensuram a dimensão nutricional ${ }^{1,4}$.

Os demais indicadores são considerados indiretos. A disponibilidade calórica permite inferir sobre a IAN quando insuficiente para suprir as necessidades dos indivíduos, porém não retrata a qualidade das calorias disponíveis. Indicadores de consumo alimentar compreendem proxy da IAN quando o de grupos alimentares e/ou nutrientes não atende as recomendações. Distrofias nutricionais e fome oculta, dadas pela avaliação antropométrica e bioquímica, permitem inferir sobre esta situação. Indicadores socioeconômicos e demográficos, característicos de pobreza e de condições de habitação inadequadas, também estão relacionados à IAN. Ressalta-se que nenhum destes indicadores isolados permite avaliar a IAN uma vez que cada um abarca vertente específica. Assim, recomenda-se a utilização de indicadores complementares para avaliação, possibilitando um diagnóstico fidedigno desta situação $0^{1,4-6}$.

Diante da complexidade de avaliação da IAN e das implicações negativas da mesma, classificando-a como problema de saúde pública ${ }^{4,5}$, torna-se necessário averiguar os indicadores que têm sido utilizados para a mensuração desta situação. Ademais, este estudo é justificado pela necessidade de compreender quais indicadores são utilizados para mensurar as dimensões desta situação multifacetada, tendo em vista que o único indicador validado, a EBIA, abarca apenas a dimensão alimentar, não refletindo as demais vertentes da IAN ${ }^{1,5}$.

Portanto, este estudo objetivou investigar sistematicamente os indicadores utilizados na avaliação da IAN e os fatores associados, em estudos nacionais.

\section{Metodologia}

Inclui-se nesta revisão sistemática artigos originais, realizados no Brasil. Não se impôs limites quanto ao ano de publicação e à unidade de estudo. Para a pesquisa dos documentos utilizouse as bases de dados SciELO, Lilacs e Medline. Optou-se pelo filtro "país/região como assunto Brasil", nas bases Lilacs e Medline, para selecionar apenas estudos nacionais, visto que estas bases apresentam elevado número de publicações internacionais. Visando incluir artigos não identificados nas bases de dados selecionadas realizou-se busca reversa nas referências dos artigos selecionados após leitura na íntegra. A etapa de busca dos artigos ocorreu de maio a agosto de 2017.

Os termos de busca utilizados foram segurança alimentar ou segurança alimentar e nutricional combinados com antropometria, estado nutricional, disponibilidade, consumo de alimentos, percepção, escala, despesa, pobreza ou renda, bem como suas respectivas traduções em inglês e espanhol. Embora nem todos sejam descritores, optou-se por estes termos considerando os indicadores de IAN propostos pela Organização das Nações Unidas para a Alimentação e a Agricultura (FAO) e os presentes em estudos sobre a temática. Assim, a pergunta norteadora desta revisão foi "Quais indicadores utilizados na avaliação da IAN no Brasil?”

O protocolo de identificação e seleção dos artigos baseou-se na leitura do título, depois do resumo e, por fim, do artigo na íntegra. A pesquisa inicial identificou 994 artigos, sendo 917 excluídos por não atenderem aos critérios de inclusão ou serem repetidos. Com a busca reversa, incluiu-se 12 novas publicações. Assim, 89 artigos compuseram esta revisão (Figura 1).

As etapas de identificação, seleção e inclusão desta revisão sistemática seguiram as recomendações PRISMA (Preferred Reporting Items for Systematic Reviews and Meta-Analysis) ${ }^{7}$, sendo realizada em pares de pesquisadores. A revisão dos artigos selecionados seguiu protocolo, elaborado pelas autoras, que direcionou a leitura visando identificar indicadores utilizados na avaliação da IAN, unidade amostral e a relação com fatores associados. Extraiu-se informações metodológicas e de resultados com o intuito de sistematização e classificação da qualidade destes manuscritos. A qualidade dos artigos elegíveis foi avaliada por protocolo Grading of Recomendations Assessment, Developing and Evaluation (GRADE), que fornece informações sobre as evidências dos artigos, considerando presença de viés, inconsistência e imprecisão de resultados, dentre outros fatores ${ }^{8,9}$. 
Figura 1. Etapas da elaboração da revisão sistemática.

\section{Resultados}

Os 89 estudos incluídos nesta revisão foram publicados no período de 2005 a 2017 e apresentaram qualidade que variou de baixa a alta, segundo os critérios de análise adotados. Entre os artigos, $15,7 \%(\mathrm{n}=14)$ foram classificados como de qualidade alta, indicando que o efeito estimado reflete o real; $32,6 \%(n=29)$ como de moderada, no qual o efeito estimado é próximo ao observado; e $51,7 \%(n=46)$ como de baixa, sugerindo que o efeito apresentado nos estudos provavelmente é diferente do real. Todos os artigos iniciaram a avaliação com pontuação mínima por serem do tipo observacional, sendo um com desenho do tipo coorte $\mathrm{e}^{10} \mathrm{e}$ os demais transversais. Os quesitos que mais contribuíram para a pontuação foram: controle por variáveis de confusão e medidas de associação e magnitude maiores que dois.

A maioria $(92,1 \%, n=82)$ avaliou a IA por escalas e perguntas referentes à percepção, sendo que $83,1 \%(\mathrm{n}=74)$ utilizaram a EBIA, $5,62 \%$ (n =5) a Escala Americana de Insegurança Alimen$\operatorname{tar}^{11-15}$, um a escala de IA validada para adolescentes $^{16} \mathrm{e}$ dois questões isoladas sobre a perda de quantidade e qualidade de alimentos ${ }^{17,18}$. Tanto 
a EBIA quanto a Escala Americana foram utilizadas em diferentes versões, variando de 5 a 18 questões para o diagnóstico da IA (Figura 2).

Os sete demais estudos utilizaram outros indicadores para verificar a IAN. Um avaliou em nível municipal, utilizando 20 indicadores disponíveis em bases de dados, contemplando as dimensões de disponibilidade, acesso e consumo de alimentos, além da utilização biológica dos nutrientes ${ }^{19}$. Dois utilizaram modelo estatístico para predição de $\mathrm{IA}^{20,21}$, segundo dados socioeconômicos e demográficos, disponíveis da Pesquisa Nacional de Amostras de Domicílios (PNAD) de 2004 e Censo Demográfico de 2010. Um estudo avaliou a IAN por meio de seis das sete dimensões desta situação propostas pelo Conselho Nacional de Segurança Alimentar e Nutricional (CONSEA $)^{22}$, sendo elas de produção de alimentos; renda, acesso e gastos com alimentos; alimentação adequada; saúde e acesso a serviços de saúde; educação e políticas públicas que promovem a SAN, a partir de dados secundários disponibilizados pelo Instituto Brasileiro de Geografia e Estatística (IBGE) e Ministérios da Saúde e do Desenvolvimento Social. A IAN também foi avaliada pela presença de desnutrição em crianças $^{23}$, ingestão insuficiente de calorias por adulto equi- valente ${ }^{24}$ e por despesa com alimentação superior a $70 \%$ da renda familiar ${ }^{25}$ (Figura 2).

A maioria dos estudos apresentou como unidade amostral famílias com presença de determinado grupo etário (crianças, adolescentes ou idosos) ou com característica específica (indígenas, quilombolas ou beneficiários do Programa Bolsa Família). Entre os estudos, 19,1\% ( $\mathrm{n}=17)$ avaliaram o domicílio sem estabelecer presença de integrante específico ${ }^{10,11,24-37}$. Apenas dois dos estudos avaliaram a IA no município ${ }^{19,20}$ e um na macrorregião Centro Oeste ${ }^{22}$ (Quadro 1).

Entre as publicações, $19,1 \%(\mathrm{n}=17)$ utilizaram banco de dados de inquéritos nacionais como a Pesquisa Nacional de Demografia e Saúde - PNDS (2006) $)^{13,14,29,38-46}$, PNAD $(2004)^{26,30,31,47}$ e Pesquisa de Orçamento Familiar - POF (19951996, 2002-2003, 2008-2009) $)^{24,25}$.

Os estudos que avaliaram a IA, segundo a EBIA, encontraram associação com fatores socioeconômicos e demográficos, consumo alimentar, estado nutricional, condições de saúde, estilo de vida, dentre outros. As associações mais presentes foram com as variáveis socioeconômicas e demográficas, principalmente renda; escolaridade, sexo e cor/raça do morador de referência; número de moradores; esgotamento sanitário;

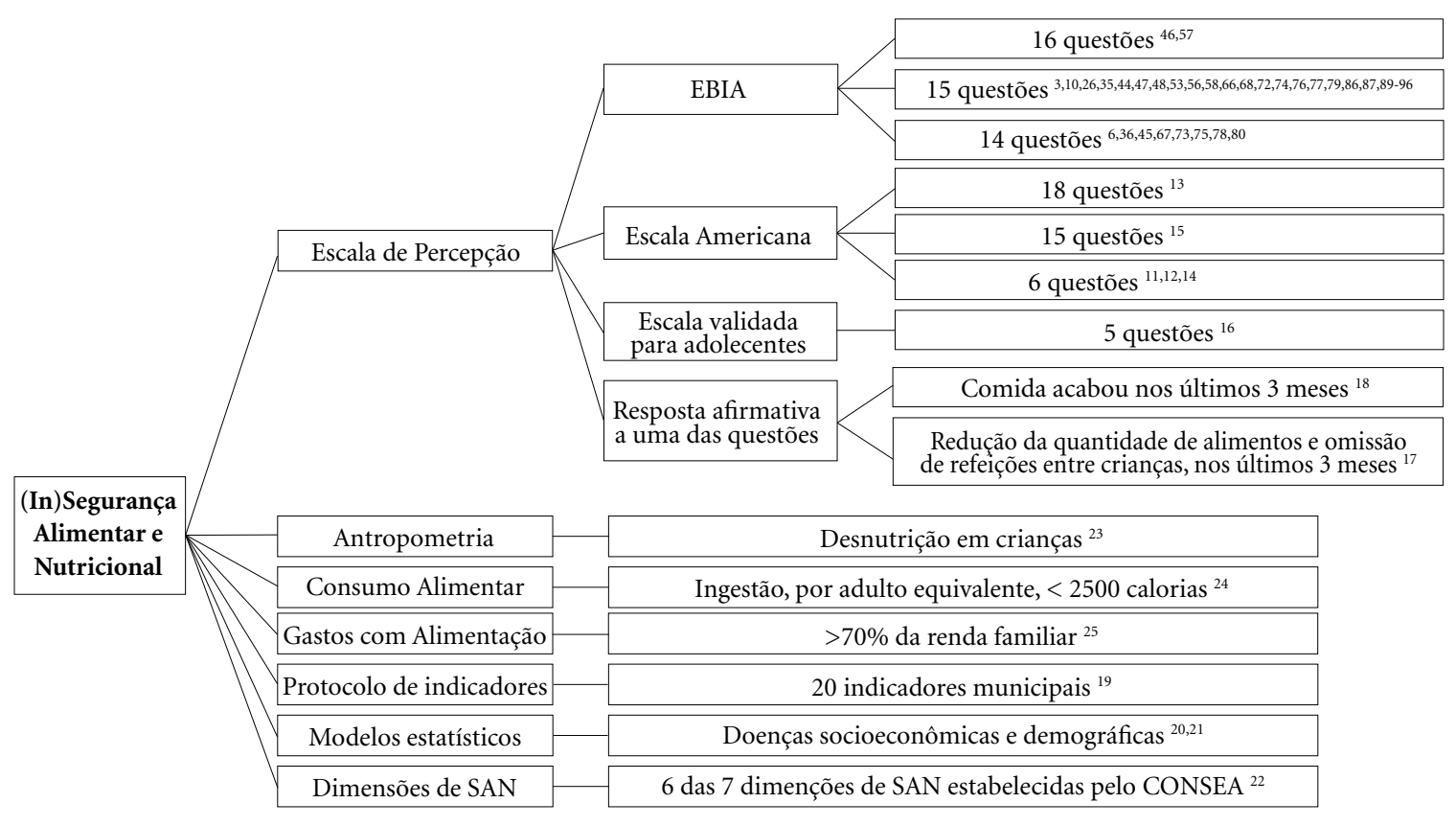

Figura 2. Indicadores de (in) segurança alimentar e nutricional utilizados em estudos brasileiros. 
Quadro 1. Indicadores e segurança alimentar e nutricional utilizados, segundo unidade amostral, em estudos brasileiros.

\begin{tabular}{|c|c|c|}
\hline $\begin{array}{l}\text { Indicador de } \\
\text { insegurança alimentar e } \\
\text { nutricional }\end{array}$ & & Unidade amostral \\
\hline \multirow{8}{*}{$\begin{array}{l}\text { Escalas e questões sobre } \\
\text { Percepção }\end{array}$} & \multirow{5}{*}{$\begin{array}{l}\text { Famílias com } \\
\text { determinada } \\
\text { característica }\end{array}$} & $\begin{array}{l}\text { Chefiadas por idosos }{ }^{47}, \text { Com idosos }^{48} \text {, Com } \\
\text { crianças }^{13,15,17,43,45,46,53,56,61,65,66,82,84,85}, \text { Com crianças e adolescentes }^{50,51,92} \text {, } \\
\text { Com adolescentes }^{16,62}, \text { Com adolescentes e adultos }\end{array}$ \\
\hline & & $\begin{array}{l}\text { Beneficiárias do Programa Bolsa Família }{ }^{57,58,60,68,76,81,93} \text {; Indígenas }{ }^{49} \text {, } \\
\text { Quilombolas }^{54,78}\end{array}$ \\
\hline & & $\begin{array}{l}\text { Cobertas pela Estratégia de Saúde da Família }{ }^{75} \text { ou Núcleo de Apoio à } \\
\text { Saúde da Família }{ }^{69} \text {, Acompanhadas pela Pastoral da Criança }{ }^{90}\end{array}$ \\
\hline & & De catadores ${ }^{14}$ e ex-catadores ${ }^{72}$ \\
\hline & & Zona rural $^{52}$, assentados rurais ${ }^{3,91}$, acampados e/ou boia-fria ${ }^{18}$ \\
\hline & \multicolumn{2}{|c|}{ Domicílios $^{10,11,27-30,33-37}$} \\
\hline & \multicolumn{2}{|c|}{$\begin{array}{l}\text { Crianças }^{26,31,38,40,41,44,60,63,70,74,80,86,87,94,95}, \text { Adolescentes }^{39,59,79}, \text { Crianças e adolescentes }^{77,89} \text {, } \\
\text { Crianças, adolescentes e mulheres }{ }^{42}, \text { Mulheres em idade reprodutível }^{6,55}, \text { Gestantes } \\
\text { que frequentam Unidade Básica de Saúde }{ }^{80}\end{array}$} \\
\hline & \multicolumn{2}{|c|}{$\begin{array}{l}\text { Frequentadores }{ }^{64,67,71} \text { e trabalhadores de restaurante } \text { popular }^{73} \text {; Portadores de HIV/ } \\
\text { AIDS atendidos no serviço público }{ }^{12}\end{array}$} \\
\hline Antropometria & \multicolumn{2}{|c|}{ Famílias de trabalhadores rurais sem-terra ${ }^{23}$} \\
\hline Consumo alimentar & \multicolumn{2}{|c|}{ Domicílios $^{24}$} \\
\hline Gastos com alimentação & \multicolumn{2}{|l|}{ Domicílios $^{25}$} \\
\hline Protocolo de indicadores & \multicolumn{2}{|l|}{ Município $^{19}$} \\
\hline Modelos estatísticos & \multicolumn{2}{|c|}{ Município $^{20}$, Domicílios $^{21}$} \\
\hline Dimensões de SAN & \multicolumn{2}{|c|}{ Macrorregião ${ }^{22}$} \\
\hline
\end{tabular}

IMC: índice de massa corporal; SAN: segurança alimentar e nutricional

participação em programa de transferência condicionada de renda; e material de construção do domicílio. Observou-se também associações com consumo de vegetais, frutas e carnes; estado nutricional, principalmente o índice estatura/idade, presença de baixo peso e excesso de peso; e fome oculta, avaliada pelos baixos níveis de hemoglobina e retinol sanguíneos (Figura 3).

A IA, avaliada pelos demais indicadores de percepção (Escala Americana de Insegurança Alimentar, Escala validada para adolescentes e questões isoladas) associou-se a escolaridade do morador de referência; número de moradores; renda familiar; presença de doenças; consumo de frutas e hortaliças regionais e disponibilidade de alimentos $^{11-13,15,16,18}$ (Figura 4).

A desnutrição em crianças, como indicativo de insegurança nutricional, associou-se a características do domicílio, peso ao nascer, produção para autoconsumo e aleitamento materno ${ }^{23}$. A IA avaliada pela despesa com alimentação associouse a escolaridade morador de referência, renda, presença de menor de 18 anos, local do domicílio e produção de alimentos ${ }^{25}$. A ingestão calórica, por adulto equivalente, como proxy de IAN, as- sociou-se à renda, escolaridade, sexo do morador de referência, presença de eletrodomésticos, localização (macrorregião) e situação (urbano e rural) do domicílio, presença de crianças, adolescentes e idosos, despesa com alimentação e número de moradores/cômodo ${ }^{24}$. E a IAN avaliada por modelo estatístico de predição associou-se à renda per capita, escolaridade, sexo e raça/cor do morador de referência, abastecimento de água, localização e situação do domicílio's (Figura 4).

A IAN não se associou a fatores socioeconômicos e demográficos, de consumo alimentar, estado nutricional, condições de saúde, estilo de vida ou a outros indicadores em 16,9\% $(n=15)$ dos estudos ${ }^{6,17,19,20,22,37,41,69,90-96}$.

Entre os artigos classificados como de alta qualidade ${ }^{3,21,31,36,43,45,50,61,62,66,67,73,75,78}$, após ajuste estatístico, observou-se maior associação e magnitude entre IA, medida pela EBIA, e modelo estatístico, e variáveis relacionadas ao chefe da família ou ao morador de referência (menor escolaridade, raça negra ou mulata, cor não branca, sexo feminino, desemprego, trabalho informal, menor variedade da dieta); mãe (cor parda, mestiça ou preta; menor escolaridade); morador específico 


\begin{tabular}{|c|c|}
\hline $\begin{array}{l}\text { Fatores } \\
\text { socioeconômicos } \\
\text { e demográficos }\end{array}$ & $\begin{array}{l}\text { Renda (familiar, per capita, pela venda o produção de alimentos); nível socioeconômico; } \\
\text { escolaridade (morador de referência, materna, moradores); sexo, idade e cor/raça (morador de } \\
\text { referência, mãe, morador); trabalho sem carteira assinada ou desemprego (morador de referência, } \\
\text { morador); número de moradores, cômodos, moradores/cômodo, moradores/dormitório, filhos, } \\
\text { crianças, morador<18 anos; presença de filhos, morador <18 anos e idosos; participação em } \\
\text { programas governamentais(transferência condicionada de renda, cesta de alimentos, garantia de } \\
\text { safra, cisternas); material de construção do domicílio; domicílio doado/ocupado; esgotamento } \\
\text { sanitário; destino das fezes; tratamento e abastecimento de água; destino do lixo, presença de } \\
\text { utensílios, eletrodomésticos e serviços (fogão, geladeira, freezer, televisão, filtro de água, vaso } \\
\text { sanitário, máquina de lavar, celular, telefone fixo,luz elétrica); dívida referente à alimentação; gasto } \\
\text { com alimentação, satisfação das necesidades básicas (moradia, saúde, transporte, alimentação, } \\
\text { vestuário); localização (macroregião) e situação do domicílio (urbano ou rural) }\end{array}$ \\
\hline $\begin{array}{l}\text { Condições de } \\
\text { saúde, estilo de } \\
\text { vida e outros }\end{array}$ & $\begin{array}{l}\text { Presença de verminoses; tabagismo (morador de referência, mãe); ordem de nascimento } \\
\text { da criança; percepção de saúde; cansaço após caminhada; presença de doenças (asma, cárie } \\
\text { dental e câncer); hospitalização no último ano; doação de alimentos; refeiçães realizadas em } \\
\text { restaurante popular; insatisfação com a composição e regularidade da alimentação; violência no } \\
\text { domicílio; visita e prestação de cuidados por profissionais de saúde; produção de alimentos para } \\
\text { autoconsumo; conhecimento sobre nutrição (em relação a fracionamento, variedade e importância } \\
\text { do consumo de frutas e verduras); preferências alimentares (em função do preço, sabor e saú- } \\
\text { de) }\end{array}$ \\
\hline $\begin{array}{l}\text { Consumo } \\
\text { alimentar }\end{array}$ & $\begin{array}{l}\text { Consumo dos alimentos: verduras, legumes, frutas, suco de frutas; carne, leite, derivados do leite, } \\
\text { cereais, raízes, pães, biscoitos, café, açúcar, doces, refrigerante, óleo, gordura, alimentos processados; } \\
\text { ingestão dos nutrientes, proteína, ferro, carboidrato, cálcio, aleitamento materno (total e exclusivo); } \\
\text { qualidade e diversidade da dieta; intervalo entre refeições; não realizar desjejum, almoço ou jan- } \\
\operatorname{tar}^{3,32,38,45,46,48,51,52,59,63,64,80,81}\end{array}$ \\
\hline $\begin{array}{c}\text { Estado } \\
\text { Nutricional } \\
\text { (Antropometria e } \\
\text { Bioquímicos) }\end{array}$ & $\begin{array}{l}\text { Z-escore dos índices estatura/idade, peso/idade, peso/estatura e IMC/idade; baixo peso (IMC/idade, } \\
\text { peso/idade, IMC); excesso de peso (IMC/idade); obesidade (IMC); perda de peso; níveis sanguíneos } \\
\text { de hemoglobina e retinol; hiperglicemia } \\
\text { 32,40-42,53,63,64,68,72,82-88 }\end{array}$ \\
\hline
\end{tabular}

Figura 3. Fatores associados a insegurança alimentar, segundo Escala Brasileira de Insegurança Alimentar, em estudos brasileiros.

(cor preta, insatisfeitos com a composição e a regularidade da alimentação). A IA também esteve relacionada a variáveis da família ou domicílio, como menor renda, recebimento de benefício governamental, maior densidade de moradores, maior número de crianças e adolescentes, material de construção do domicílio não de alvenaria, não abastecimento de água pela rede pública, domicílio localizado nas macrorregiões Norte, Nordeste ou Centro Oeste, e com menor qualidade e diversidade da dieta, entre outros (Figuras 3 e 4).

Segundo os indicadores utilizados, 96,6\% (n = 86) dos estudos avaliaram apenas a dimensão alimentar da insegurança enquanto um a nutricional $^{23}$. Apenas dois dos artigos abarcaram as vertentes alimentar e nutricional da insegurança, por protocolo de indicadores referentes às dimensões de disponibilidade e acesso aos alimentos e utilização biológica dos nutrientes ${ }^{19}$ e pela avaliação das dimensões de IAN, proposta pelo CONSEA $^{22}$.

\section{Discussão}

Os indicadores utilizados na avaliação da IAN refletem os determinantes desta situação, como o acesso aos alimentos, calculado pelas escalas e questões de percepção, ingestão calórica e gastos com a alimentação, avaliando as consequências da mesma, como a presença de desnutrição em crianças e consumo alimentar inadequado ${ }^{1,97,98}$. 


\begin{tabular}{|c|c|}
\hline $\begin{array}{l}\text { Indicadores de } \\
\text { (in)segurança } \\
\text { alimentar e } \\
\text { nutricional }\end{array}$ & Fatores associados \\
\hline $\begin{array}{l}\text { Escala Americana } \\
\text { de Insegurança } \\
\text { Alimentar }\end{array}$ & $\begin{array}{l}\text { Fatores socioeconômicos: a escolaridade do morador de referência, número de moradores, } \\
\text { renda familiar }{ }^{11,12} \\
\text { Condições de saúde, estilo de vida e outros: tosse e diarreia na criança, cárie dental }{ }^{13,15}\end{array}$ \\
\hline $\begin{array}{l}\text { Escala validada } \\
\text { para adolescentes }\end{array}$ & $\begin{array}{l}\text { Condições de saúde, estilo de vida e outros: tipo de escola frequentada }{ }^{16} \\
\text { Consumo alimentar: consumo de hortaliças e frutas regionais }{ }^{16}\end{array}$ \\
\hline Questões isoladas & Condições de saúde, estilo de vida e outros: disponibilidade de alimentos ${ }^{18}$ \\
\hline Antropometria & $\begin{array}{l}\text { Fatores socioeconômicos e demográficos: tipo de moradia, idade da criança, banheiro } \\
\text { dentro do domicílio e presença de eletrodomésticos (fogão a gás, geladeira e freezer), } \\
\text { número de cômodos }{ }^{23} \\
\text { Condições de saúde, estilo de vida e outros: peso ao nascer, produção para autoconsumo de } \\
\text { carne (boi e frango), leite e ovo } \\
\text { Consumo alimentar: aleitamento materno }\end{array}$ \\
\hline $\begin{array}{c}\text { Gastos com } \\
\text { Alimentação }\end{array}$ & $\begin{array}{l}\text { Fatores socioeconômicos e demográficos: escolaridade, morador de referência, renda, } \\
\text { presença < } 18 \text { anos, localização do domicílio (macrorregião) } \\
\text { Condições de saúde, estilo de vida e outros: produção de grãos, verduras e legumes }{ }^{25}\end{array}$ \\
\hline $\begin{array}{l}\text { Consumo } \\
\text { Alimentar }\end{array}$ & $\begin{array}{c}\text { Fatores socioeconômicos e demográficos: renda, escolaridade dos moradores, sexo do } \\
\text { morador de referência, presença de eletrodomésticos (fogão a gás, geladeira), localização e } \\
\text { situação (zona urbana ou rural) do domicílio, presença de crianças, adolescentes e idosos, } \\
\text { despesa com alimentação, número de moradores/cômodos }{ }^{24}\end{array}$ \\
\hline Modelo Estatístico & $\begin{array}{l}\text { Fatores socioeconômicos e demográficos: renda per capita; escolaridade, sexo e raça/cor do } \\
\text { morador de referência; abasteciento de água, localização e situação do domicílio }{ }^{21}\end{array}$ \\
\hline
\end{tabular}

Figura 4. Fatores associados a insegurança alimentar e nutricional, segundo diferentes indicadores, em estudos brasileiros.

Ressalta-se que nenhum dos estudos avaliou a IA pela disponibilidade calórica per capita, que quando menor que o somatório das necessidades energéticas de todos os integrantes da família ou menor que 2.500 calorias per capita indicam IAN. A avaliação da disponibilidade calórica é um método recomendado pela FAO e utilizado na avaliação e no monitoramento dos países ${ }^{1,499}$.

A EBIA é validada para a população brasileira e deve ser preferencialmente utilizada em relação às escalas internacionais ou adaptadas, podendo ser aplicada em populações urbanas, rurais e específicas, como indígenas e quilombolas. Entretanto, observou-se que a Escala Americana de In- segurança Alimentar continua sendo utilizada em estudos brasileiros. Além disso, verificou-se a utilização de escala de IA validada para adolescentes brasileiros. Em relação às adaptações, a EBIA foi adaptada, em termos de linguagem, para famílias indígenas ${ }^{49}$, com a justificativa da peculiaridade desta população; e aplicada na forma original em famílias quilombolas ${ }^{78}$. A utilização de diferentes instrumentos dificulta a comparação dos resultados encontrados, porém, em alguns casos, faz-se necessárias adaptações em função da população de estudo, a fim de compreender as especificidades e contribuir com a caracterização mais próxima da situação de IAN vivenciada. 
Foram utilizadas diferentes versões da EBIA, variando de 14 a 16 itens. Ressalta-se a recomendação, desde 2014, de aplicação da EBIA contendo 14 questões, visando aprimorar a escala para a realidade de transição nutricional vivenciada no país ${ }^{100}$.

A utilização de questões isoladas sobre a percepção da quantidade dos alimentos é pouco relatada na literatura. Porém, questões que abordam a redução da quantidade de alimentos consumida retratam seu menor acesso, presente nas escalas nacional e internacionais, podendo remeter a um proxy de IA. A utilização de versões reduzidas de escalas de percepção tem sido apresentada como alternativa de mensuração da situação de IA, visando menores custos de aplicação, principalmente em estudos populacionais ${ }^{101}$. Indagar sobre a perda de quantidade dos alimentos é uma situação muito específica da IAN, pois vai além da preocupação com a privação do alimento, presente nas escalas de IA.

O protocolo de indicadores de IAN mostrouse instrumento útil para avaliar esta situação, em nível municipal. Como principal vantagem temse a utilização conjunta de indicadores de distintas dimensões, permitindo visão mais ampla da situação e utilização de bases de dados disponíveis. Destaca-se que este protocolo permitiu classificar o município em IAN leve, moderada ou grave, segundo as dimensões de disponibilidade e acesso, consumo alimentar e utilização biológica de nutrientes, respectivamente ${ }^{19}$.

Os modelos estatísticos de predição também são considerados relevantes na avaliação da IAN por permitirem utilização de múltiplos indicadores. Porém, os apresentados nesta revisão, abarcaram apenas a dimensão alimentar da insegurança em função dos indicadores utilizados.

A IAN, avaliada pela proposta do CONSEA, engloba: dimensões de disponibilidade, acesso, utilização e estabilidade por meio dos indicadores de produção e consumo de alimentos; despesa com alimentação; renda per capita; distribuição de macronutrientes; IA, segundo a EBIA; estado nutricional de crianças menores de cinco anos, adolescentes e adultos; baixo peso ao nascer; taxa de mortalidade infantil; aleitamento materno exclusivo; acesso ao pré-natal; alimentos contaminados por agrotóxicos; abastecimento de água, serviço de coleta de lixo e esgotamento sanitário. Embora a avaliação acima contemple todas as dimensões da IAN, esta é realizada isoladamente por dimensão, de forma descritiva, sem análise conjunta dos múltiplos indicadores e dimensões.

A avaliação antropométrica tem sido utilizada como medida proxy de IAN, na dimensão de utilização biológica. O indicador de IAN pela presença de desnutrição em crianças permite detecta-la no seu nível mais grave, ou seja, quando a perda de qualidade e quantidade de alimentos na família já atingiu as crianças ${ }^{23}$. Além disso, reflete a redução dos alimentos em quantidade e qualidade. Por não ser um indicador sensível, e também por sofrer influência de outros determinantes que não só a IA, deve ser utilizado com cautela, sendo complementado por outros indicadores $^{4,5}$.

A IAN avaliada pela ingestão insuficiente de calorias, por adulto equivalente, retrata a suficiência quantitativa dos alimentos, sem considerar a qualidade dos mesmos, implicando na necessidade de outros métodos em conjunto. Apresenta como vantagem a ponderação do consumo dentro do domicílio, considerando a presença de crianças, e não apenas a informação de um único integrante da família ${ }^{24,99}$.

Já o indicador de despesas com a alimentação, quando há alto gasto com estas pela família, demonstra o comprometimento das outras necessidades essenciais, prejudicando a SAN ${ }^{1,25}$. Porém, este método não considera doações e trocas de alimentos, e se há produção de alimentos para autoconsumo ${ }^{99}$.

Observou-se, entre as unidades de estudo, a utilização do termo 'família' quando avaliaram algum integrante ou característica específica e de 'domicílio' quando analisaram características do morador de referência e do próprio domicílio. Estes termos são próximos, mas não são considerados sinônimos, uma vez que a família é compreendida como conjunto de pessoas ligadas por laços de parentesco, dependência doméstica ou normas de convivência, que compartilham o mesmo domicílio. Já domicílio é o local de moradia de uma pessoa ou de um grupo, com laços de parentesco, dependência doméstica ou normas de convivência ${ }^{102}$.

Entre os estudos incluídos, verificou-se a utilização do indicador de IAN para o domicílio ou para um ou mais integrantes da família, principalmente o morador de referência, também chamado de chefe da família. Nenhum artigo avaliou todos os membros da família, porém uma das publicações utilizou a metodologia de adulto equivalente que pondera o consumo de calorias para os demais integrantes da família ${ }^{24,99}$.

Ainda em relação à unidade amostral, verificou-se que quatro estudos utilizaram banco de dados da PNAD edição 2004, o qual contém bloco exclusivo de avaliação de segurança alimentar pela EBIA. Este inquérito apresenta variáveis 
socioeconômicas e demográficas que podem ser relacionadas à IA, mas não contêm informações sobre a dimensão nutricional desta situação. Ressalta-se que nenhum dos estudos utilizou as versões mais recentes da PNAD, realizadas em 2009 e em 2013.

A PNDS, realizada em 2006, foi a pesquisa mais utilizada, entre os artigos avaliados, sendo a IA detectada pela EBIA. Esta pesquisa permite relacionar a IA com variáveis de estado nutricional, consumo alimentar, fatores socioeconômicos e demográficos e de saúde de mulheres de 15 a 49 anos e crianças até cinco anos, mas não contem informações sobre os demais integrantes do domicílio. Os bancos de dados da POF também foram utilizados para avaliação da IA, tendo sido empregadas as metodologias de ingestão calórica por adulto equivalente e a de despesa da família com a alimentação.

Ressalta-se que embora a POF não apresente bloco específico sobre IAN, a utilização da mesma na avaliação deste fenômeno é de grande valia, pois apresenta informações sobre indicadores socioeconômicos e demográficos, disponibilidade alimentar, antropometria e consumo alimentar de maiores de 10 anos, que podem complementar esta análise. A POF realizada em 2008-2009 é a única pesquisa de representatividade nacional que apresenta banco de dados capaz de contemplar as distintas dimensões da IAN. Destaca-se que há previsão de inclusão da EBIA a partir da próxima POF, cuja coleta foi iniciada em 2017, fato este que ampliará a discussão sobre a temática.

A utilização de inquéritos nacionais para a avaliação da IAN é relevante, pois são representativos da população brasileira, permitindo estratificar os resultados segundo localização e situação do domicílio, condições socioeconômicas, entre outras características de interesse ${ }^{103}$. Além disso, a POF apresenta bloco sobre condições de vida, com questão relativa à percepção do informante sobre a suficiência da quantidade de alimento consumida $^{102}$, que pode ser utilizada como proxy de IA, desde que complementada com outros indicadores presentes na pesquisa. Ampliar a utilização dos inquéritos para a avaliação da situação de IAN expandirá os achados deste fenômeno.

Embora já estabelecida, a necessidade de utilização complementar de indicadores para avaliação da IAN faz com que a maioria dos pesquisadores utilize apenas um para diagnóstico, $\mathrm{o}$ associando com outros, representantes de outras dimensões. Este fato pode ser explicado pela ausência, até o momento, de metodologia específica que permita esta avaliação conjunta. Ressalta-se que a FAO recomenda a criação de protocolo padrão de indicadores da IAN, desde 1996, com o intuito de contemplar as distintas vertentes desta situação e ser utilizado na avaliação em todos os níveis (micro, meso e macro) $)^{2,5,104}$.

No Brasil, a EBIA é o único instrumento validado para a avaliação da IA, porém não contempla as demais vertentes, além do acesso aos alimentos. Visando melhor compreensão da IAN, em suas diferentes dimensões, alguns autores propõem protocolos ou modelos estatísticos de predição, porém estes têm sido utilizados de forma pontual. Um dos estudos incluídos ${ }^{32}$, embora tenha avaliado a IA pela EBIA, analisou a situação por meio de modelo de equações estruturais, contendo indicadores socioeconômicos, antropométricos, de consumo alimentar, de produção para autoconsumo e de recebimento de Bolsa Família. Esta análise permite verificar os fatores mais relacionados com a IA, por meio de cargas fatoriais, possibilitando a avaliação da situação de forma mais abrangente.

Para os estudos que apresentam variáveis socioeconômicas, de estado nutricional, de consumo e de disponibilidade alimentar, tem-se a possibilidade de fazer inferências sobre a situação de IAN a partir destes indicadores, como situação de pobreza, distrofia nutricional, inadequação no consumo e na disponibilidade. Estes não são considerados medidas diretas de IAN, mas o podem ser como proxy da mesma, sendo utilizados de forma complementar na avaliação desta situação.

Fazer inferências sobre a situação de IAN, utilizando indicadores das dimensões alimentar e nutricional, é uma decisão complexa em função dos vários fatores relacionados a esta situação, que vão desde a renda insuficiente até o reflexo destas privações no estado nutricional e na instauração da fome oculta ${ }^{18}$. Mesmo diante da complexidade, deve-se investir em metodologias capazes de avaliar de forma ampla e sensível a IAN 2,5 .

Embora cerca da metade dos estudos incluídos nesta revisão não sejam classificados como de qualidade moderada à alta, os fatores socioeconômicos, demográficos, de consumo alimentar e de distrofia nutricional também foram observados nos estudos considerados de maior confiabilidade. Ressalta-se que existem outros protocolos de avaliação da qualidade e que os artigos poderiam receber classificações diferentes da apresentada, caso fosse utilizado um outro. Em função da ausência de consenso para avaliação da qualidade dos manuscritos publicados, optou-se 
por não excluir desta revisão os não classificados como de alta qualidade, visando contemplar todos os artigos que respondam à questão central desta revisão, sobre quais indicadores são utilizados na avaliação da IA.

A qualidade desta revisão foi avaliada pelo critério Assessment of Multiple Systematic Reviews $(\text { AMSTAR })^{105}$, sendo que esta não contemplou o quesito relacionado à inclusão de literatura cinzenta (dissertações, teses, livros e relatórios) na busca sistemática. Esta limitação foi atenuada pela inclusão de alguns materiais com estas características na discussão do manuscrito.

\section{Conclusão}

Apenas um dos estudos incluídos nesta revisão avaliou a insegurança pelas dimensões alimentar e nutricional, utilizando protocolo com indica- dores que contemplam estas vertentes. Os demais avaliaram a dimensão alimentar ou nutricional, segundo indicadores isolados ou agrupados em modelos estatísticos e verificaram fatores associados à situação de insegurança, mas sem utiliza -los de forma complementar.

A qualidade de cerca da metade dos estudos incluídos foi considerada de moderada à alta, permitindo estabelecer relação entre a IAN e fatores socioeconômicos, demográficos, de consumo alimentar e de estado nutricional. Os estudos que apresentaram maior qualidade utilizaram a EBIA e modelo estatístico de predição na avaliação da IA.

É necessário maior aprofundamento metodológico para a elaboração de instrumento de avaliação de IAN, por meio de indicadores complementares das múltiplas facetas da insegurança, que contemple as dimensões de disponibilidade, acesso, utilização e estabilidade, no Brasil.

\section{Colaboradores}

DC Morais e SO Lopes trabalharam na concepção, redação e revisão crítica do manuscrito. SE Priore revisou criticamente o manuscrito e aprovou a versão final do mesmo. 


\section{Referências}

1. Kepple AW, Segall-Correa AM. Conceituando e medindo segurança alimentar e nutricional. Cien Saude Colet 2011; 16(1):187-199.

2. Leroy JL, Ruel M, Frongillo EA, Harris J, Ballard TJ. Measuring the Food Access Dimension of Food Security: A Critical Review and Mapping of Indicators. Food Nutr Bull 2015; 36(2):167-195.

3. Almeida JA, Santos AS, Nascimento MAO, Oliveira JVC, Silva DG, Mendes-Netto RS. Fatores associados ao risco de insegurança alimentar e nutricional em famílias de assentamentos rurais. Cien Saude Colet 2017; 22(2):479-488.

4. Jones AD, Ngure FM, Pelto G, Young SL. What Are We Assessing When We Measure Food Security? A Compendium and Review of Current Metrics. Adv Nutr 2013; 4(5):481-505.

5. Kepple AW. O estado da segurança alimentar e nutricional no Brasil - Um retrato multidimensional. Roma: FAO; 2014.

6. Pinheiro MM, Oliveira JS, Leal VS, Lira PPC, Souza NP, Campos FACS. Prevalência do excesso de peso e fatores associados em mulheres em idade reprodutiva no Nordeste do Brasil. Rev. Nutr. 2016; 29(5):679-689.

7. Moher D, Liberati A, Tetzlaff J, Altman DG; PRISMA Group. Preferred Reporting Items for Systematic Reviews and Meta-Analyses: The PRISMA Statement. Ann Intern Med 2009; 151(4):264-270.

8. Guyatt G, Oxman AD, Akl EA, Kunz R, Vist G, Brozek J, Norris S, Falck-Ytter Y, Glasziou P, DeBeer H, Jaeschke R, Rind D, Meerpohl J, Dahm P, Schünemann HJ. GRADE guidelines: 1. Introductiond GRADE evidence profiles and summary of findings tables. J Clin Epidemiol 2011; 64(4):383-394.

9. Galvão TF, Pereira MG. Avaliação da qualidade da evidência de revisões sistemáticas. Epidemiol. Serv. Saúde 2015; 24(1):173-175.

10. Cabral CS, Lopes AG, Lopes JM, Vianna RPT. Segurança alimentar, renda e Programa Bolsa Família: estudo de coorte em municípios do interior da Paraíba, Brasil, 2005-2011. Cad Saude Publica 2014; 30(2):393402.

11. Santos JV, Gigante DP, Domingues MR. Prevalência de insegurança alimentar em Pelotas, Rio Grande do Sul, Brasil, e estado nutricional de indivíduos que vivem nessa condição. Cad Saude Publica 2010; 26(1):41-49.

12. Charão APS, Batista MHRS, Ferreira LB. Food insecurity of HIV/AIDS patients at a unit of outpatient healthcare system in Brasilia, Federal District, Brazil. Rev. Soc. Bras. Med. Trop. 2012; 45(6):751-753.

13. Reis M. Food insecurity and the relationship between Household income and children's health and Nutrition in Brazil. Health Econ 2012; 21(4):405-427.

14. Santos LMP, Carneiro FF, Hoefel MGL, Santos W, Nogueira TQ. The precarious livelihood in waste dumps: A report on food insecurity and hunger among recyclable waste collectors. Rev. Nutr. 2013; 26(3):323-334.

15. Frazão P, Benício MHD, Narvai PC, Cardoso MA. Food insecurity and dental caries in schoolchildren: a cross-sectional survey in the western Brazilian Amazon. Eur J Oral Sci 2014; 122(3):210-215.

16. Coelho SEAC, Gubert MB. Insegurança alimentar e sua associação com consumo de alimentos regionais brasileiros. Rev. Nutr. 2015; 28(5):555-567.
17. Carvalho Filho EV, Aquino JS, Vianna RPT. Insegurança e Consumo Alimentar no Município de Princesa Isabel-Paraíba-Brasil. Rev. Bras. Ciências da Saúde 2006; 10(3):277-286.

18. Carneiro FF, Tambellini AT, Silva JA, Haddad JPA, Búrigo AC, Sá WR, Viana FC, Bertolini VA. Saúde de famílias do Movimento dos Trabalhadores Sem Terra e de bóias-frias, Brasil, 2005. Rev Saude Publica 2008; 42(4):757-763.

19. Panelli-Martins BE, Santos SMC, Assis AMO. Segurança alimentar e nutricional: desenvolvimento de indicadores e experimentação em um município da Bahia, Brasil. Rev. Nutr. 2008; 21(Supl.):65-81.

20. Gubert MB, Benício MHD, Santos LMP. Estimativas de insegurança alimentar grave nos municípios brasileiros. Cad Saude Publica 2010; 26(8):1595-1605.

21. Gubert MB, Benício MH, Silva JP, Costa Rosa TE, Santos SM, Santos LM. Use of a predictive model for food insecurity estimates in Brazil. Arch. Latinoam. Nutr. 2010; 60(2):119-125.

22. Tinoco SGG, Mendes JFR, Figueiredo AC, Costa APR, Leão MM, Santos LM. Segurança Alimentar e Nutricional na Região Centro-Oeste: particularidades e contrastes. Segurança Alimentar e Nutricional 2011; 18(1):58-72.

23. Lang RMF, Almeida CCBA, Taddei JAAC. Segurança alimentar e nutricional de crianças menores de dois anos de famílias de trabalhadores rurais Sem Terra. Cien Saude Colet 2011; 16(7):3111-3118.

24. Costa LV, Silva MMC, Braga MJ, Lírio VS. Fatores associados à segurança alimentar nos domicílios brasileiros em 2009. Economia e Sociedade 2014; 23(2):373394.

25. Costa LV, Gomes MFM Lírio VS, Braga MJ. Produtividade Agrícola e Segurança Alimentar dos Domicílios das Regiões Metropolitanas Brasileiras. RESR 2013; 51(4):661-680.

26. Hofmann R. Determinantes da Insegurança Alimentar no Brasil: Análise dos Dados da PNAD de 2004. Segurança Alimentar e Nutricional 2008; 15(1):49-61.

27. Salles-Costa R, Pereira RA, Vasconcellos MTL, Veiga GV, Marins VMR, Jardim BC, Gomes FS, Sichieri R. Associação entre fatores socioeconômicos e insegurança alimentar: estudo de base populacional na Região Metropolitana do Rio de Janeiro, Brasil. Rev. Nutr. 2008; 21(Supl.):99-109.

28. Vianna RPT, Segall-Corrêa AM. Insegurança alimentar das famílias residentes em municípios do interior do estado da Paraíba, Brasil. Rev. Nutr. 2008; 21(Supl.):111-122.

29. Gubert MB, Santos LMP. Determinantes da insegurança alimentar no Distrito Federal. Com. Ciências Saúde 2009; 20(2):143-150.

30. Marin-Leon L, Francisco PMSB, Segall-Correa AM, Panigassi G. Bens de consumo e insegurança alimentar: diferenças de gênero, cor de pele autorreferida e condição socioeconômica. Rev Bras Epidemiol 2011; 14(3):398-410.

31. Mondini L, Rosa TE, Gubert MB, Sato GS, Benício MHA. Insegurança alimentar e fatores sociodemográficos associados nas áreas urbana e rural do Brasil. Informações Econômicas 2011; 41(2):52-60. 
32. Oliveira LDS, Lima-Filho DO. Modelo de segurança alimentar e nutricional e seus determinantes socioeconômicos e comportamentais. Cad. Gestão Pública e Cidadania 2011; 16(59):240-259.

33. Ferreira HS, Souza MECA, Moura FA, Horta BL. Prevalência e fatores associados à Insegurança Alimentar e Nutricional em famílias dos municípios do norte de Alagoas, Brasil, 2010. Cien Saude Colet 2014; 19(5):1533-1542.

34. Interlenghi GS, Rosana Salles-Costa R. Inverse association between social support and household food insecurity in a metropolitan area of Rio de Janeiro, Brazil. Public Health Nutr 2014; 18(16):2925-2933.

35. Ribeiro-Silva RC, Fiaccone RL, Barreto ML, Santana MLP, Santos SMC, Conceição-Machado MEP, Aliaga MA. The association between intimate partner domestic violence and the food security status of poor families in Brazil. Public Health Nutr 2015; 19(7):1305-1311.

36. Souza BFNJ, Marin-Leon L, Camargo DFM, SegallCorrea AM. Demographic and socioeconomic conditions associated with food insecurity in households in Campinas, SP, Brazil. Rev. Nutr. 2016; 29(6):845-857.

37. Rodrigues LPF, Carvalho RC, Maciel A, Otanasio PN, Garavello MEPE, Nardoto GB. Food Insecurity in Urban and Rural Areas in Central Brazil: Transition from Locally Produced Foods to Processed Items. Ecology of Food and Nutrition 2016; 55(4):365-377.

38. Gomes GP, Gubert MB. Aleitamento materno em crianças menores de 2 anos e situação domiciliar quanto à segurança alimentar e nutricional. J Pediatr 2012; 88(3):279-282.

39. Kac G, Velásquez-Melendez G, Schlüssel MM, Segall-Côrrea AM, Silva AA, Pérez-Escamilla R. Severe food insecurity is associated with obesity among Brazilian adolescent females. Public Health Nutr 2012; 15(10):1854-1860.

40. Kac G, Schlüssel MM, Pérez-Escamilla R, VelásquezMelendez G, Silva AAM. Household Food Insecurity Is Not Associated with BMI for Age or Weight for Height among Brazilian Children Aged 0-60 Months. PLoS ONE 2012; 7(9):e45747.

41. Santos LP, Gigante DP. Relação entre insegurança alimentar e estado nutricional de crianças brasileiras menores de cinco anos. Rev Bras Epidemiol 2013; 16(4):984-994.

42. Schlüssel MM, Silva AAM, Pérez-Escamilla R, Kac G. Household food insecurity and excess weight/obesity among Brazilian women and children: a life-course approach. Cad Saude Publica 2013; 29(2):219-241.

43. Poblacion AP, Marín-León L, Segall-Corrêa AM, Silveira JA, Taddei JAAC. Insegurança alimentar em domicílios brasileiros com crianças menores de cinco anos. Cad Saude Publica 2014; 30(5):1067-1078.

44. Vega JB, Taddei JAAC, Poblacion AP. Características sociodemográficas e nutricionais de crianças brasileiras menores de 2 anos beneficiárias de programas de transferência condicionada de renda em 2006. Cien Saude Colet 2014; 19(3):931-942.

45. Bortolini GA, Vitolo MR, Gubert MB, Santos LMP. Iniquidades sociais influenciam a qualidade e a diversidade da dieta de crianças brasileiras de 6 a 36 meses. Cad Saude Publica 2015; 31(11):2413-2424.
46. Poblacion AP, Cook JT, Marín-León L, Segall-Corrêa AM, Silveira JAC, Konstantyner T, Taddei JAAC. Food Insecurity and the Negative Impact on Brazilian Children's Health-Why Does Food Security Matter for Our Future Prosperity? Brazilian National Survey (PNDS 2006/07). Food and Nutrition Bulletin 2016; 37(4):585-598.

47. Rosa TEC, Mondini L, Gubert MB, Sato GS, Benício MHA. Segurança alimentar em domicílios chefiados por idosos, Brasil. Rev. Bras. Geriatr. Gerontol. 2012; 15(1):69-77.

48. Marín-León L, Segal-Corrêa AM, Panigassi G, Maranha LK, Sampaio MAFA, Pérez-Escamilla R. A percepção de insegurança alimentar em famílias com idosos em Campinas, São Paulo, Brasil. Cad Saude Publica 2005; 21(5):1433-1440.

49. Fávaro T, Ribas DLB, Zorzatto JR, Segall-Corrêa AM, Panigassi G. Segurança alimentar em famílias indígenas Teréna, Mato Grosso do Sul, Brasil. Cad Saude Publica 2007; 23(4):785-793.

50. Panigassi G, Segall-Corrêa AM, Marin-León L, Pérez -Escamilla R, Sampaio MFA, Maranha LK. Insegurança alimentar como indicador de iniquidade: análise de inquérito populacional. Cad Saude Publica 2008; 24(10):2376-2384.

51. Panigassi G, Segall-Corrêa AM, Marin-León L, PérezEscamilla R, Maranha LK, Sampaio MFA. Insegurança alimentar intrafamiliar e perfil de consumo de alimentos. Rev. Nutr. 2008; 21(Supl.):135-144.

52. Nobre LN, Murta NMG, Souza MM, Ferreira NC, Cardoso LM, Hamacek FR. Segurança Alimentar em uma Comunidade Rural no Alto Vale do Jequitinhonha/MG. Segurança Alimentar e Nutricional 2009; 16(1):18-31.

53. Pimentel PG, Sichieri R, Salles-Costa R. Insegurança alimentar, condições socioeconômicas e indicadores antropométricos em crianças da Região Metropolitana do Rio de Janeiro/Brasil. R. Bras. Est. Pop. 2009; 26(2):283-294.

54. Monego ET, Peixoto MRG, Cordeiro MM, Costa RM. (In) segurança alimentar de comunidades quilombolas do Tocantins. Segurança Alimentar e Nutricional 2010; 17(1):37-47.

55. Velasquez-Melendez G, Schlüssel MM, Brito AS, Silva AAM, Lopes-Filho JD, Kac G. Mild but Not Light or Severe Food Insecurity is Associated with Obesity among Brazilian Women. J. Nutr. 2011; 141(5):898902.

56. Aires JS, Martins MC, Joventino ES, Ximenes LB. (In) Segurança alimentar em famílias de pré-escolares de uma zona rural do Ceará. Acta Paul Enferm 2012; 25(1):102-108.

57. Anschau FR, Matsuo T, Segall-Corrêa AM. Insegurança alimentar entre beneficiários de programas de transferência de renda. Rev. Nutr. 2012; 25(2):177189.

58. Dias MM, Machado HM, Ferreira CS, Oliveira VL, Gomes AP, Cantaluppi ETC. Situação de insegurança alimentar de famílias beneficiárias de programas de transferência de renda na unidade de saúde da família comunidade São João Baptista, Petrópolis/Rio de Janeiro. Rev APS 2012; 15(2):199-205. 
61. Bittencourt LS, Santos SMC, Pinto EJ, Aliaga MA, Ribeiro-Silva RC. Factors Associated with Food Insecurity in Households of Public School Students of Salvador City, Bahia, Brazil. Health Popul Nutr 2013; 31(4):471-479.

62. Guerra LDS, Espinosa MM, Bezerra ACD, Guimarães LV, Lima-Lopes MA. Insegurança alimentar em domicílios com adolescentes da Amazônia Legal Brasileira: prevalência e fatores associados. Cad Saude Publica 2013; 29(2):335-348.

63. Pedraza DF, Queiroz D, Menezes TN. Segurança alimentar em famílias com crianças matriculadas em creches públicas do Estado da Paraíba, Brasil. Rev. Nutr. 2013; 26(5):517-527.

64. Souza BFNJ, Letícia Marín-León L. Food insecurity among the elderly: Cross-sectional study with soup kitchen users. Rev. Nutr. 2013; 26(6):679-691.

65. Aquino JS, Sequeira-de-Andrade LAS, Silva PEBA, Silva AP, Vieira CRS, Lira PIC. Food insecurity and socioeconomic, food and nutrition profile of schoolchildren living in urban and rural areas of Picos, Piauí. Rev. Nutr. 2014; 27(4):395-404.

66. Facchini LA, Nunes BP, Motta JVS, Tomasi E, Silva SM, Thumé E, Silveira DS, Siqueira FV, Dilélio AS, Saes MO, Miranda VIA, Volz PM, Osório A, Fassa AG. Insegurança alimentar no Nordeste e Sul do Brasil: magnitude, fatores associados e padrões de renda per capita para redução das iniquidades. Cad Saude Publica 2014; 30(1):161-174.

67. Godoy KC, Sávio KEO, Akutsu RC, Gubert MB, Botelho RBA. Perfil e situação de insegurança alimentar dos usuários dos Restaurantes Populares no Brasil. Cad Saude Publica 2014; 30(6):1239-1249.

68. Monteiro F, Schmidt ST, Costa IB, Almeida CCB, Matuda NS. Bolsa Família: insegurança alimentar e nutricional de crianças menores de cinco anos. Cien Saude Colet 2014; 19(5):1347-1357.

69. Peixoto MRG, Ramos K, Martins KA, Schincaglia RM, Braudes-Silva LA. Insegurança alimentar na área de abrangência do Núcleo de Apoio à Saúde da Família em Itumbiara, Goiás. Epidemiol. Serv. Saúde 2014; 23(2):327-336

70. Rocha EMB, Lima RT, Almeida PC. Insegurança alimentar relacionada à área de residência em município do Semiárido brasileiro. Cad. Saúde Colet. 2014; 22(2):205-211.

71. Martins Sobrinho F, Silva YC, Abreu MNS, Pereira SCL, Dias Júnior CS. Fatores determinantes da insegurança alimentar e nutricional: estudo realizado em Restaurantes Populares de Belo Horizonte, Minas Gerais, Brasil. Cien Saude Colet 2014; 19(5):1601-1611.

72. Bezerra TA, Figueroa-Pedraza D. (In)segurança alimentar entre famílias com crianças menores de cinco anos residentes em área de vulnerabilidade social de Campina Grande, Paraíba. Rev. Nutr. 2015; 28(6):655665.

73. Falcão ACML, Aguiar OB, Fonseca MJM. Association of socioeconomic, labor and health variables related to Food Insecurity in workers of the Popular Restaurants in the city of Rio de Janeiro. Rev. Nutr. 2015; 28(1):77-87.

74. Figueroa-Pedraza D, Gama JSFA. Segurança alimentar e nutricional de famílias com crianças menores de cinco anos do município de Campina Grande, Paraíba. Rev Bras Epidemiol 2015; 18(4):906-917.

75. Sabóia RCB, Santos MM. Prevalência de insegurança alimentar e fatores associados em domicílios cobertos pela Estratégia Saúde da Família em Teresina, Piauí, 2012-2013. Epidemiol. Serv. Saúde 2015; 24(3):749758.

76. Sperandio N, Priore SE. Prevalência de insegurança alimentar domiciliar e fatores associados em famílias com pré-escolares, beneficiárias do Programa Bolsa Família em Viçosa, Minas Gerais, Brasil. Epidemiol. Serv. Saúde 2015; 24(4):739-748.

77. Barros KCS, Hipólito TLB, Bezerra MGS, Rocha CVS, Oliveira ES, Santos LFS, Freire JAP. Perfil Socioeconômico e Nutricional de Escolares em (In)Segurança Alimentar de uma Região do Semiárido Nordestino. Rev. Bras. Ciências da Saúde 2016; 20(1):5-10.

78. Gubert MB, Segall-Corrêa AM, Spaniol AM, Pedroso J, Coelho SEAC, Pérez-Escamilla R. Household food insecurity in black-slaves descendant communities in Brazil: has the legacy of slavery truly ended? Public Health Nutr 2016; 20(8):1513-1522.

79. Santim GC, Pintarelli TP, Fraiz FC, Oliveira ACB, Paiva SM, Ferreira FM. Association between untreated dental caries and household food insecurity in schoolchildren. Cien Saude Colet 2016; 21(2):573-584.

80. Antunes MML, Sichieri R, Salles-Costa R. Consumo alimentar de crianças menores de três anos residentes em área de alta prevalência de insegurança alimentar domiciliar. Cad Saude Publica 2010; 26(8):1642-1650.

81. Lignani JB, Sichieri R, Burlandy L, Salles-Costa R. Changes in food consumption among the Programa Bolsa Familia participant families in Brazil. Public Health Nutrition 2010; 14(5):785-792.

82. Oliveira JS, Lira PIC, Andrade SLLS, Sales AC, Maia SR, Batista Filho M. Insegurança Alimentar e estado nutricional de crianças de São João do Tigre, no semi-árido do Nordeste. Rev Bras Epidemiol 2009; 12(3):413-423.

83. Oliveira JS, Lira PIC, Veras ICL in memorian; Maia SR, Lemos MCC, Andrade SLLS, Viana Junior MJ, Pinto FCL, Leal VS, Batista Filho M. Estado nutricional e insegurança alimentar de adolescentes e adultos em duas localidades de baixo índice de desenvolvimento humano. Rev. Nutr. 2009; 22(4):453-465.

84. Oliveira JS, Lira PIC, Osório MM, Sequeira LAS, Costa EC, Gonçalves FCLSP, Batista Filho M. Anemia, hipovitaminose A e insegurança alimentar em crianças de municípios de Baixo Índice de Desenvolvimento Humano do Nordeste do Brasil. Rev Bras Epidemiol 2010; 13(4):651-664. 
85. Oliveira JS, Lira PIC, Maia SR, Sequeira LAS, Amorim RCA, Batista Filho M. Insegurança alimentar e estado nutricional de crianças de Gameleira, zona da mata do Nordeste brasileiro. Rev. Bras. Saúde Matern. Infant. 2010; 10(2):237-245.

86. Vieira VL, Souza JMP, Cervato-Mancuso AM. Insegurança alimentar, vínculo mãe-filho e desnutrição infantil em área de alta vulnerabilidade social. Rev. Bras. Saúde Matern. Infant. 2010; 10(2):199-207.

87. Vicenzi K, Henn RL, Weber AP, Backes V, Paniz VMV, Donatti T, Olinto MTA. Insegurança alimentar e excesso de peso em escolares do primeiro ano do Ensino Fundamental da rede municipal de São Leopoldo, Rio Grande do Sul, Brasil. Cad Saude Publica 2015; 31(5):1084-1094.

88. Oliveira ACM, Tavares MCM, Bezerra AR. Insegurança alimentar em gestantes da rede pública de saúde de uma capital do nordeste brasileiro. Cien Saude Colet 2017; 22(2):519-526.

89. Ribeiro-Silva RC, Oliveira-Assis AM, Junqueira SB, Fiaccone RL, Santos SMC, Barreto ML, Pinto EJ, Silva LA, Rodrigues LC, Alcantara-Neves NM. Food and nutrition insecurity: a marker of vulnerability toasthma symptoms. Public Health Nutr 2013; 17(1):14-19.

90. Vieira VL, Fiore EG, Cervato-Mancuso AM. Insegurança Alimentar em Região de Alta Vulnerabilidade Social da Cidade de São Paulo. Segurança Alimentar e Nutricional 2006; 13(2):34-42.

91. Busato MA, Gallina LS, Dreyer DC, Quadros JC, Lavratti E, Teo CRPA. Segurança alimentar e nutricional e as condições do ambiente em assentamento rural de Santa Catarina. Alim. Nutr. 2011; 22(4):555-559.

92. Cordeiro MM, Monego ET, Martins KA. Overweight in Goiás' quilombola students and food insecurity in their families. Rev. Nutr. 2014; 27(4):405-412.

93. Nunes TS, Cruz JMG, Pinho L. Avaliação da segurança alimentar e nutricional de famílias beneficiárias do Programa Bolsa Família. Nutrire 2014; 39(2):233-242.

94. Figueroa Pedraza D, Queiroz D, Paiva AA, Cunha MAL, Lima ZN. Seguridad alimentaria, crecimiento y niveles de vitamina $\mathrm{A}$, hemoglobina y zinc en niños preescolares del nordeste de Brasil. Cien Saude Colet 2014; 19(2):641-650.

95. Lisboa MBMC, Oliveira EO, Lamounier JA, Silva CAM, Freitas RN. Prevalence of iron-deficiency anemia in children aged less than 60 months: A population-based study from the state of Minas Gerais, Brazil. Rev. Nutr. 2015; 28(2):121-131.

96. Souza MM, Figueroa Pedraza D, Menezes TN. Estado nutricional de crianças assistidas em creches e situação de (in)segurança alimentar de suas famílias. Cien Saude Colet 2012; 17(12):3425-3436.
97. Pérez-Escamilla R, Segall-Corrêa AM. Food insecurity measurement and indicators. Rev. Nutr. 2008; 21(Supl.):15-26.

98. Morais DC, Dutra LV, Franceschini SCC, Priore SE. Insegurança alimentar e indicadores antropométricos, dietéticos e sociais em estudos brasileiros: uma revisão sistemática. Cien Saude Colet 2014; 19(5):1475-1488.

99. Smith LC, Subandoro A. Measuring food security using household expenditure surveys. Food security in practice technical guide series. Washington: International food policy research institute; 2007.

100. Segall-Corrêa AM, Marin-León L, Melgar-Quiñonez $\mathrm{H}$, Pérez-Escamilla R. Refinement of the Brazilian Household Food Insecurity Measurement Scale: Recommendation for a 14-item EBIA. Rev. Nutr. 2014; 27(2):241-251.

101. Santos LP, Lindemann IL, Motta JVS, Mintem G, Bender E, Gigante DP. Proposta de versão curta da Escala Brasileira de Insegurança Alimentar. Rev Saude Publica 2014; 48(5):783-789.

102. Instituto Brasileiro de Geografia e Estatísticas (IBGE). Pesquisa de Orçamentos Familiares 2008-2009. Despesas, rendimentos e condições de vida. Rio de Janeiro: IBGE; 2010.

103. Sperandio N, Priore SE. Inquéritos antropométricos e alimentares na população brasileira: importante fonte de dados para o desenvolvimento de pesquisas. Cien Saude Colet 2017; 22(2):499-508.

104. Organización de las Naciones Unidas para la Agricultura y la Alimentación (FAO). Declaración de Roma sobre la Seguridad Alimentaria Mundial. Cumbre Mundial sobre la alimentación. Roma: FAO; 1996.

105. Shea BJ, Jeremy M Grimshaw JM, Wells GA, Boers M, Andersson N, Hamel C, Porter AC, Tugwell P, Moher D, Bouter LM. Development of AMSTAR: a measurement tool to assess the methodological quality of systematic reviews. BMC Medical Research Methodology 2007; 7(10):1-7.

Artigo apresentado em 09/11/2017

Aprovado em 26/11/2018

Versão final apresentada em 28/11/2018 